\title{
To find the prevalence of glaucoma in patients with diabetes mellitus attending tertiary care hospital, east Sikkim
}

\author{
Karma L. Bhutia ${ }^{1, *}$, Simran Dhakal'2, Sonam C. Bhutia ${ }^{3}$ \\ ${ }^{1}$ Associate Professor, ${ }^{2}$ MBBS Student, ${ }^{3}$ Assistant Professor, ${ }^{1}$ Dept. of Ophthalmology, ${ }^{3}$ Dept. of Biochemistry Sikkim Manipal \\ Institute of Medical Sciences, Gangtok, Sikkim
}

*Corresponding Author: Karma L. Bhutia

Email: drkarma07@gmail.com

\begin{abstract}
Aim: To find the prevalence of glaucoma in diabetic patients attending tertiary care hospital, east Sikkim.

Materials and Methods: A hospital based study of 75 diabetic patients attending the eye opd at Crh from March to August 2018 underwent complete ocular examination for glaucoma after taking a prior consent from the patient.

Results: Out of 75 diabetic patients $15(20 \%)$ of them had glaucoma. Out of 15 diabetic patients with glaucoma $73.3 \%$ were males and $26.6 \%$ were females. The mean age of diabetics females having glaucoma is $53.75 \pm 16.39$ and male is $60.36 \pm 12.14$. As far as Ethnic distribution was concerned in our patients with glaucoma $60 \%$ were Nepali, $33.3 \%$ were Bhutia and $6.7 \%$ belonged to other communities.

Conclusion: This study highlights the prevalence and the demographic characteristics of glaucoma among diabetes mellitus patients in a tertiary care hospital in east Sikkim. The northeast of India records the highest prevalence of diabetes mellitus hence the ocular manifestations of the disease are common. Since no such study has been done in Sikkim before this will play an integral role in raising awareness amongst the people about the ocular manifestations of the disease.
\end{abstract}

Keywords: Diabetes mellitus, Glaucoma, Sikkim.

\section{Introduction}

Diabetes mellitus is a syndrome of impaired carbohydrate, fat and protein metabolism caused either due to the lack of insulin secretion or decreased sensitivity of the of the tissues to insulin.

World Health Organisation (WHO) has declared that the incidence of diabetes is increasing rapidly world-wide which has become a major public health concern. ${ }^{1}$ Global prevalence of diabetes was estimated to be $2.8 \%$ in 2000 and is predicted to be $4.4 \%$ in $2030 .^{1}$

Sikkim is a small Himalayan state situated in north eastern part of India with a population of 610,577 . The incidence of diabetes mellitus is increasing in an alarming rate and of which glaucoma diagnosis is often ignored among the diabetic population. Till date there has been no published report on the proportion of diabetic patients suffering from glaucoma.

Glaucoma is a group of ocular disorders characterised by damaged to the optic nerve. In its early stages, it may present with few or no symptoms but can gradually steal sight without warning due to increased intra ocular pressure the drainage system gets blocked and the fluid cannot exit at a normal rate, thus leading to blindness if not treated timely. The increased pressure pushes against the optic nerve which may result in vision loss usually starting with peripheral or side vision. ${ }^{2}$

Drainage of aqueous humour gets obstructed which causes increased intra ocular pressure. It may be: 1) Congenital - due to developmental anomalies 2) Secondary- due to complications of other diseases 3) Primary- (a) Open angle (b) Angle closure. ${ }^{3}$
Uncontrolled Type 1 diabetes mellitus or Type 2 diabetes mellitus for a long enough period will lead to the development of diabetic retinopathy, which then triggers new blood vessel formation in the ocular anterior segment and interferes with the normal internal drainage of the eye leading to glaucoma. There is considerable evidence that $\mathrm{T} 2$ diabetes mellitus is a risk factor for primary open angle glaucoma. ${ }^{4}$

Glaucoma is a gradually advancing optic neuropathy which may lead to permanent blindness affecting 66.8 million people worldwide in year 2000 . In India, prevalence of glaucoma range from $4.96 \%$ to $14.6 \%$. The World Health Organization (WHO) has reported glaucoma as important eye disease affecting 66.8 million people throughout the world whose treatment has to be done as soon as possible. According to vision 2020 initiative, glaucoma is significantly contributing to global blindness percentage. The risk has been reported to be 1.6-4.7 times higher in individuals with diabetes than in non diabetic

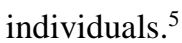

\section{Review of Literature}

According to Sheetal Dharmadhikari et al the mean age was $53.8 \pm 10.7$ years. There were $320(38 \%)$ females. The prevalence of glaucoma was $15.6 \%$ (95\% CI: $13.1-18.1), 42.4 \%$ were pure vegetarians. ${ }^{6}$

According to Thakuria Jayantia et $\mathrm{al}^{7}$ the mean age of glaucoma patients was 53.50 years, hereditary preponderance was found in $23.81 \%$ patients and POAG was found in $8.25 \%, 32$ out of 388 diabetics. POAG was diagnosed in 84 diabetic patients $(7.0 \%)$ in the age group of $15-75$ years. ${ }^{7}$ 
Becker et al. ${ }^{8}$ had found that $26 \%$ of the POAG patients had a positive family history of glaucoma.

According to Beena $\mathrm{R}$ et al. ${ }^{9}$ Primary open angle glaucoma was found to be the most common type of glaucoma with a prevalence of $5.8 \%$

According to Lingam Vijaya et al. ${ }^{10}$ the mean age was $53.78 \pm 10.71$ years, and $55.1 \%$ were women. Data also suggests that the prevalence of POAG varies from race to race with a significant increase in prevalence with age, but there was no difference in age- adjusted specific rates between genders.

\section{Objectives}

1. To study the proportion of glaucoma in diabetes mellitus patients.

2. To study the demographic characteristics (age group, gender, occupation, diet, weight, ethnicity) in patients having glaucoma.

3. To find out whether glaucoma is inherited.

\section{Materials and Methods}

Study Design: This was a hospital based crosssectional study conducted in the department of Ophthalmology in Central Referral Hospital (CRH), Gangtok.

Study Population: 75 diabetic patients visiting the Eye opd were taken.

Study Duration: The study was carried out for 6 months from March- August 2018.

A total of 75 patients with diabetes mellitus (DM) attending the eye out patient department (OPD) were selected with approval from the Institutional Ethics Committee (IEC).

Fasting and post prandial blood sugar level were recorded for the confirmation of diabetes mellitus along with the clinical history.

Patients blood pressure was measured and recorded.

Detailed questionnaire was prepared for the patients and written informed consent was obtained from each patients prior to the study. The questionnaire included questions regarding age, sex, race, occupation, diet, family history and the knowledge of diabetes and glaucoma.

Inclusion Criteria: Patients with 18 years of age and above, diagnosed with diabetes mellitus, following the standard diagnostic criteria recommended by American Diabetic Association. ${ }^{6}$

Exclusion Criteria: Any active eye diseases like conjunctivitis, iridocylitis.

A comprehensive eye examination was done by an ophthalmologist including measurement of IOP by applanation tonometry, evaluating the drainage angle of the eye by doing gonioscopy. The optic disc examination by direct ophthalmoscopy and a visual field perimetry was done for each patient.
Statistical Analysis was done by using the Statistical Package for Social Sciences. ${ }^{7}$ Data has been represented as percentages, mean \pm standard deviation.

\section{Results}

A total of 75 diabetic patients participated in the study, out of that 50 were males 25 were females. The mean age of the participants was $56.69 \pm 13.4$. The mean age of diabetic females having glaucoma is 53.75 \pm 16.39 and male is $60.36 \pm 12.14$. Number of years for which diabetes mellitus was present in the glaucoma patients was $9.73 \pm 6.75$ years.

$77.3 \%$ of the patients were non vegetarians and $22.7 \%$ were vegetarians. All the glaucoma patients $(100 \%)$ were non vegetarians.

On observation of the hereditary characteristics, none of the patients reported a positive family history of glaucoma.

Only 1 patient had previous knowledge about the relationship of glaucoma and eye problems.

Table 1: The distribution of male and female in the study population $(n=75)$

\begin{tabular}{|l|c|c|}
\hline \multicolumn{1}{|c|}{ Sex } & Frequency & Percentage \\
\hline Male & 50 & 66.7 \\
\hline Female & 25 & 33.3 \\
\hline
\end{tabular}

$66.7 \%$ of the study population were males and $33.3 \%$ were females

Table 1.1: Distribution of glaucoma patients in study population $(n=75)$

\begin{tabular}{|l|c|c|}
\hline $\begin{array}{c}\text { Presence of } \\
\text { Glaucoma }\end{array}$ & Frequency & Percentage \\
\hline Yes & 15 & 20 \\
\hline No & 60 & 80 \\
\hline
\end{tabular}

Out of 75 diabetic patients who visited the Ophthalmology department $20 \%$ of them had glaucoma.

Table 1.2: Distribution of glaucoma based on Sex $(\mathrm{n}=15)$

\begin{tabular}{|l|c|c|}
\hline \multicolumn{1}{|c|}{ Sex } & Frequency & Percentage \\
\hline Male & 11 & 73.3 \\
\hline Female & 4 & 26.6 \\
\hline
\end{tabular}

Out of the diabetic patients with glaucoma $73.3 \%$ were males and $26.6 \%$ were females.

Table 1.3: Frequency of distribution of glaucoma based on ethnicity $(n=15)$

\begin{tabular}{|l|c|c|}
\hline Ethnicity & Frequency & Percentage \\
\hline Nepali & 9 & 60 \\
\hline Bhutia & 5 & 33.3 \\
\hline Others & 1 & 6.7 \\
\hline
\end{tabular}


Out of 15 diabetic patients having glaucoma $60 \%$ were Nepali, $33.3 \%$ were Bhutia and $6.7 \%$ belonged to other non local communities. Glaucoma was not observed in the Lepcha population.

\section{Discussion}

Our study showed that among 75 diabetes mellitus patients attending the Ophthalmology department in a Tertiary Care hospital in east Sikkim $20 \%$ had glaucoma. Many of the diabetic patients had visited the eye opd for routine examination to get the fundus checked for diabetic retinopathy.

The mean age of the subjects was $56.69 \pm 13.4$ which is quite similar to the results in a research done by Sheetal Dharmadhikari et al where the mean age was $53.8 \pm 10.7$ years. $^{8}$ The total percentage of females was $33.38 \%$ whereas in their study the percentage of females was $38 \%$, the prevalence of glaucoma was found to be $15.6 \%$ whereas in our study the prevalence was found to be $20 \%$ and the same study showed that $42.4 \%$ of the glaucoma patients were vegetarians whereas in our study all the patients were nonvegetarians. ${ }^{8}$ The fact that all the patients were non vegetarians can be owed to the socio-cultural factors and food habits of the Sikkimese people. In another study done in the north east the prevalence was only found to be $8.25 \% .{ }^{9}$ hereditary preponderance in the mentioned study was $23.81 \%$ whereas in our study there was no such positive family history of glaucoma. ${ }^{9}$ Becker et al. ${ }^{10}$ had found that $26 \%$ of the POAG patients had a positive family history of glaucoma but in our study none of the patients reported a positive family history. According to Beena R et al. ${ }^{11}$ Primary open angle glaucoma was found to be the most common type of glaucoma with a prevalence of 5.8\%, similarly in our study all the glaucoma patients had primary open angle glaucoma.

\section{Conclusion}

This study highlights the prevalence and the demographic characteristics of Glaucoma among diabetes mellitus patients in a tertiary care hospital in east Sikkim. The northeast of India records the highest prevalence of diabetes mellitus hence the ocular manifestations of the disease are common. Hence this study would document the prevalence of glaucoma in diabetes mellitus patients in Sikkim where no such study has been done before and play an integral role in raising awareness amongst the people.

\section{Reference}

1. The World Health Organization. Geneva: 2001. World Health Report: Mental Health: New understanding New Hope. The institute.

2. Quigley HA, Broman AT. The number of people with glaucoma worldwide in 2010 and 2020. Br J Ophthalmol. 2006;90(3):262-7.

3. Deepthi S, Gopal B. Prevalence of different types of glaucoma in type II diabetics and non-diabetics- A comparative study. International Journal of Current Medical Research. 2015;4(6):379-80.

4. Wild S, Roglic G, Green A, Sicree R, King H. Global prevalence of diabetes: estimates for the year 2000 and projections for 2030. Diabetes Care. 2004;27.

5. American Diabetes Association. Standards of medical care in diabetes - 2015. Diabetes Care. 2015;38:S1-S9.

6. Statistical Packages for Social Sciences Version 16.0 SPSS Inc. Chicago, IL USA.

7. Dharmadhikari S, Lohiya K, Chelkar V, Kalyani VK, Dole K, Deshpande M, Khandekar R, Kulkarni S. Magnitude and determinants of glaucoma in type II diabetics: A hospital based cross-sectional study in Maharashtra, India. Oman Journal of Ophthalmology. 2015;8(1).

8. Jayanta T, Dipali DC, Santana S. Prevalence of Glaucoma Amongst Diabetic Patients Attending a Tertiary Health Care in North Eastern India.

9. Becker B, Roth FD, Kolker AE. Glaucoma Family Study. Am J Ophthalmol. 1960;50(4):557-567.

10. Beena R, Thiruvengada Senthilkumar G, Raja R. Incidence of Glaucoma \& Diabetic Retinopathy in Patients with Diabetes Mellitus in A Teaching Hospital. Journal of Medical and Dental Science Research. 2017;4(5):31-5.

How to cite this article: Bhutia K. L, Dhakal S, Bhutia S. C. To find the prevalence of glaucoma in patients with diabetes mellitus attending tertiary care hospital, east Sikkim. Indian J Clin Exp Ophthalmol. 2018;4(4):447-449. 